\title{
CONVERGENCE OF RANDOM PROCESSES WITHOUT DISCONTINUITIES OF THE SECOND KIND AND LIMIT THEOREMS FOR SUMS OF INDEPENDENT RANDOM VARIABLES
}

BY

\author{
L. Š. GRINBLAT
}

\begin{abstract}
Let $\xi_{1}(t), \ldots, \xi_{n}(t), \ldots$ and $\xi(t)$ be random processes on the interval $[0,1]$, without discontinuities of the second kind. A. V. Skorohod has given necessary and sufficient conditions under which the distribution of $f\left(\xi_{n}(t)\right)$ converges to the distribution of $f(\xi(t))$ as $n \rightarrow \infty$ for any functional $f$ continuous in the Skorohod metric. In the following we shall consider only stochastically right-continuous processes without discontinuities of the second kind, i.e., processes such that the space $X$ of their sample functions is the space of all right-continuous functions $x(t)$ $(0<t<1)$ without discontinuities of the second kind. For a set $T=$ $\left\{t_{1}, \ldots t_{n}, \ldots\right\} \subset[0,1]$ the metric $\rho_{T}$ is defined on $X$ as in 2.3. The metric $\rho_{T}$ defines on the $X$ the minimal topology in which all functionals continuous in Skorohod's metric and also the functionals $x\left(t_{1}-0\right)$, $x\left(t_{1}\right), \ldots, x\left(t_{n}-0\right), x\left(t_{n}\right), \ldots$ are continuous. We will give necessary and sufficient conditions under which the distribution of $f\left(\xi_{n}(t)\right)$ converges to the distribution of $f(\xi(t))$ as $n \rightarrow \infty$ for any completely continuous functional $f$, i.e. for any functional $f$ which is continuous in any of the metrics $\rho_{T}$ defined in 2.3 .
\end{abstract}

1. Introduction. The study of random processes with independent increments is of special interest. Let us formulate one of the basic results of the present paper. Let $\xi(\omega)$ be a random variable defined on the probability space $\Omega$. For any $d>0$ we set

$$
\xi^{(d)}(\omega)=\left\{\begin{array}{lll}
0 & \text { if } & |\xi(\omega)|<d, \\
\xi^{2}(\omega) & \text { if } & |\xi(\omega)|>d .
\end{array}\right.
$$

THEOREM 4. Let $\xi_{1}(t), \ldots, \xi_{n}(t), \ldots$ and $\xi(t)$ be random processes on the interval $[0,1]$ with independent increments and without discontinuities of the second kind, such that for any $\varepsilon>0$ there exists $d>0$ such that $E \xi_{n}^{(d)}(t)<\varepsilon$ for all $n$ and $t$. For the distribution of $f\left(\xi_{n}(t)\right)$ to converge to the distribution of $f(\xi(t))$ as $n \rightarrow \infty$ for any completely continuous functional $f$, it is necessary and sufficient that the following conditions be satisfied:

Received by the editors November 6, 1975.

AMS (MOS) subject classifications (1970). Primary 60B10, 60G50.

O American Mathematical Society 1977 
(a) For any finite set $t_{1}, \ldots, t_{k} \subset[0,1]$ the joint distribution of

$$
\xi_{n}\left(t_{1}-0\right), \xi_{n}\left(t_{1}\right), \ldots, \xi_{n}\left(t_{k}-0\right), \xi_{n}\left(t_{k}\right)
$$

converges to the joint distribution of

$$
\xi\left(t_{1}-0\right), \xi\left(t_{1}\right), \ldots, \xi\left(t_{k}-0\right), \xi\left(t_{k}\right)
$$

as $n \rightarrow \infty$;

(b) The sequence of functions $\varphi_{n}(t)=E \xi_{n}(t)$ converges uniformly on the interval $[0,1]$.

Let us assume that for the processes $\xi_{1}(t), \ldots, \xi_{n}(t), \ldots$ and $\xi(t)$ of Theorem 4 there exists an at most countable set $T \subset[0,1]$ such that the sample functions of these processes have no discontinuities at points in $[0,1] \backslash T$ with probability 1 . It follows from Theorem 4 (see 3.4 ) that the conditions (a) and (b) of Theorem 4 are necessary and sufficient for the distribution of $f\left(\xi_{n}(t)\right)$ to converge to the distribution of $f(\xi(t))$ as $n \rightarrow \infty$ for any functional $f$ continuous in the uniform metric $\rho_{c}\left(\rho_{c}\left(x_{1}, x_{2}\right)=\sup _{t} \mid x_{1}(t)\right.$ $\left.-x_{2}(t) \mid\right)$.

As an immediate consequence of the limit theorems for random processes with independent increments and without discontinuities of the second kind, one obtains limit theorems for sums of independent random variables (see §). Let us consider a simple example. Let $\xi_{1}, \ldots, \xi_{n}, \ldots$ be a sequence of independent identically distributed random variables for which $E \xi_{n}=0$, $D \xi_{n}=1$. Let us consider a random process $\eta_{n}(t)$ on the interval $[0,1]$, which equals $S_{k} / \sqrt{n}$ if $t \in[(k-1) / n, k / n)$ and $\eta_{n}(1)=S_{n} / \sqrt{n}$; the random variable $S_{k}=\xi_{1}+\cdots+\xi_{k}$. The random processes $\eta_{1}(t), \ldots \eta_{n}(t), \ldots$ and $w(t)$, where $w(t)$ is the process of Brownian motion, satisfy all the hypotheses of Theorem 4. All sample functions of these processes are continuous at irrational points with probability 1 . From the above we obtain Donsker's theorem: For any functional $f$ continuous in the uniform metric the distribution of $f\left(\eta_{n}(t)\right)$ converges to the distribution of $f(w(t))$ as $n \rightarrow \infty$.

\section{Convergence of random processes without discontinuities of the second kind.}

2.1. Let $X$ denote the set of all functions $x(t)$ on $[0,1]$ which have no discontinuities of the second kind. For the sake of simplicity, we shall assume that $x(t)$ is right-hand continuous, that is, $x(t+0)=x(t)$. In the uniform metric

$$
\rho_{c}\left(x_{1}, x_{2}\right)=\sup _{t}\left|x_{1}(t)-x_{2}(t)\right|
$$

the space $X$ is not separable. This fact forces us to consider the Skorohod metric on $X$. Let $\Lambda$ denote the set of all continuous increasing functions $\lambda(t)$ on $[0,1]$ such that $\lambda(0)=0, \lambda(1)=1$. The Skorohod metric is defined by the 
formula

$$
\rho_{s}\left(x_{1}, x_{2}\right)=\operatorname{int}_{\lambda \in \Lambda}\left[\sup _{t}\left|x_{1}(\lambda(t))-x_{2}(t)\right|+\sup _{t}|\lambda(t)-t|\right] .
$$

It is evident that the space $X$ is separable in the metric $\rho_{s}$ (see $[2, \mathrm{VI}, \S 5]$ ).

2.2. By definition a random process $\xi(t)$ on $[0,1]$ has no discontinuities of the second kind if the sample function of this process belongs to the space $X$ with probability 1 (because of this we assume from the beginning that the process $\xi(t)$ is stochastically right-hand continuous, that is, $\xi(t+0)=\xi(t))$.

For every positive number $c<1$ consider the functional

$$
\begin{aligned}
\Delta_{c}(x)= & \sup _{t-c<t^{\prime}<t<t^{\prime \prime}<t+c}\left[\min \left\{\left|x\left(t^{\prime}\right)-x(t)\right| ;\left|x(t)-x\left(t^{\prime \prime}\right)\right|\right\}\right] \\
& +\sup _{0<t<c}|x(0)-x(t)|+\sup _{1-c<t<1}|x(t)-x(1)|
\end{aligned}
$$

on $X$. In [2, VI, §5] the following theorem is proved.

THEOREM 1. Let $\xi_{1}(t), \ldots, \xi_{n}(t), \ldots$ and $\xi(t)$ be random processes without discontinuities of the second kind defined on $[0,1]$ and suppose that for any finite set of points $t_{1}, \ldots, t_{k} \subset[0,1]$ the joint distribution of

$$
\xi_{n}\left(t_{1}\right), \ldots, \xi_{n}\left(t_{k}\right)
$$

converges to the joint distribution of

$$
\xi\left(t_{1}\right), \ldots, \xi\left(t_{k}\right)
$$

as $n \rightarrow \infty$. Then the distribution of $f\left(\xi_{n}(t)\right)$ converges to the distribution of $f(\xi(t))$ as $n \rightarrow \infty$ for every functional $f$ continuous in the Skorohod metric if and only if

$$
\lim _{c \rightarrow 0} \varlimsup_{n \rightarrow \infty} P\left\{\Delta_{c}\left(\xi_{n}(t)\right)>\varepsilon\right\}=0
$$

for every $\varepsilon>0$.

2.3. There exist interesting sets open in the uniform metric which are not open in the Skorohod metric. Let $a(t), b(t) \subset X$ and $\inf _{t}[b(t)-a(t)]>0$. We set

$$
U_{a(t)}^{b(t)}=\left\{x(t): \inf _{t}[x(t)-a(t)]>0, \inf _{t}[b(t)-x(t)]>0\right\} .
$$

It is easy to prove that for the set $U_{a(t)}^{b(t)}$ to be open in the Skorohod metric it is necessary and sufficient that $a(t)$ and $b(t)$ be continuous functions.

Let $T=\left(t_{1}, \ldots, t_{n}, \ldots\right)$ be an at most countable set on $[0,1]$. Consider the metric

$$
\begin{aligned}
\rho_{T}\left(x_{1}, x_{2}\right)= & \rho_{s}\left(x_{1}, x_{2}\right) \\
& +\sum_{n=1}^{\infty} \frac{1}{2^{n}}\left[\left|x_{1}\left(t_{n}-0\right)-x_{2}\left(t_{n}-0\right)\right|+\left|x_{1}\left(t_{n}\right)-x_{2}\left(t_{n}\right)\right|\right] \text { on } X .
\end{aligned}
$$


Let us point out some simple properties of the metric $\rho_{T}$. In this metric the space $X$ is separable. A functional which is continuous in the Skorohod metric $\rho_{s}$ is also continuous in the metric $\rho_{T}$. Functionals $f_{t_{0}}^{-}(x)=x\left(t_{0}-0\right)$ and $f_{t_{0}}(x)=x\left(t_{0}\right)$, where $0<t_{0}<1$, are continuous in the metric $\rho_{T}$ if and only if $t_{0} \in T$. On the other hand, the functionals $f_{t_{0}}^{-}(x), f_{t_{0}}(x)$ are discontinuous in the metric $\rho_{s}$.

LEMMA 1. For the set $U_{a(t)}^{b(t)}$ to be open in the metric $\rho_{T}$ it is necessary and sufficient that all points of discontinuity of the functions $a(t)$ and $b(t)$ belong to $T$.

Proof. The necessity follows immediately from the definition of the metric $\rho_{T}$.

Sufficiency. For every function $x(t) \in U_{a(t)}^{b(t)}$ there exist two functions $\tilde{a}(t), \tilde{b}(t) \subset X$, which have discontinuities only at a finite number of points belonging to the set $T$ and

$$
\tilde{a}(t) \geqslant a(t), \quad \tilde{b}(t) \leqslant b(t), \quad \inf _{t}[\tilde{b}(t)-\tilde{a}(t)]>0, \quad x(t) \in U_{\tilde{a}(t)}^{\tilde{b}(t)} .
$$

It is easy to see that the set $U_{\tilde{a}(t)}^{\vec{b}(t)}$ is open in the metric $\rho_{T}$. From this it follows that the set $U_{a(t)}^{b(t)}$ is open in the metric $\rho_{T}$. This completes the proof of Lemma 1.

2.4. Definition 1. A set $V \subset X$ is said to be a domain if $V=\cup_{i=1}^{\infty} U_{a_{i}(t)}^{b_{i}(t)}$. The closure of $V$ in the Skorohod metric said to be a domain closure and is denoted by $\bar{V}$.

In particular, $U_{a(t)}^{b(t)}$ is a domain, the closure of which is the set $\bar{U}_{a(t)}^{b(t)}=$ $\{x(t): a(t) \leqslant x(t) \leqslant b(t)\}$. In 2.2 the functional $\Delta_{c}(x)$ was considered. Let $V_{c, \varepsilon}=\left\{x: \Delta_{c}(x)>\varepsilon\right\}$.

LEMMA 2. The set $V_{c, e}$ is a domain.

Proof. Let $x_{0}(t) \in V_{c, e^{-}}$This means that there exist five points $t_{1}, t_{2}, t_{3}, t_{4}, t_{5} \subset[0,1]$ which satisfy the following conditions:

$$
\begin{gathered}
t_{1} \leqslant c, t_{5}>1-c, t_{2}<t_{3}<t_{4}, t_{3}-t_{2}<c, t_{4}-t_{3}<c ; \\
\left|x_{0}(0)-x_{0}\left(t_{1}\right)\right|+\min \left[\left|x_{0}\left(t_{2}\right)-x_{0}\left(t_{3}\right)\right| ;\left|x_{0}\left(t_{3}\right)-x_{0}\left(t_{4}\right)\right|\right] \\
\quad+\left|x_{0}\left(t_{5}\right)-x_{0}(1)\right|=\varepsilon_{0}>\varepsilon .
\end{gathered}
$$

Let us assume that the function $x_{0}(t)$ is continuous at the points $t_{2}, t_{3}, t_{4}, t_{5}$ (if $t_{1}<c$ then we shall assume that the function $x_{0}(t)$ is also continuous at the point $\left.t_{1}\right)$. Consider two functions $a(t), b(t) \subset X$ such that

$$
\begin{gathered}
\inf _{t}[b(t)-a(t)]>0, \quad x_{0}(t) \in U_{a(t)}^{b(t)}, \\
x_{0}\left(t_{i}\right)-a\left(t_{i}\right) \leqslant \frac{\varepsilon_{0}-\varepsilon}{12}, \quad b\left(t_{i}\right)-x_{0}\left(t_{i}\right)<\frac{\varepsilon_{0}-\varepsilon}{12},
\end{gathered}
$$


where $t_{i}=0, t_{1}, t_{2}, t_{3}, t_{4}, t_{5}, 1$. If $t_{1}=c$ then $a(t)$ and $b(t)$ can have discontinuities only at the point $c$ (if the function $x_{0}(t)$ is continuous at $c$, then $a(t)$ and $b(t)$ are continuous functions). If $t_{1}<c$ then $a(t)$ and $b(t)$ are continuous functions. If $\hat{x}(t) \in U_{a(t)}^{b(t)}$ then $\left|\hat{x}(0)-\hat{x}\left(t_{1}\right)\right|+\min \left[\mid \hat{x}\left(t_{2}\right)-\right.$ $\left.\hat{x}\left(t_{3}\right)|;| \hat{x}\left(t_{3}\right)-\hat{x}\left(t_{4}\right) \mid\right]+\left|\hat{x}\left(t_{5}\right)-\hat{x}(1)\right| \geqslant\left(\varepsilon_{0}+\varepsilon\right) / 2>\varepsilon$. Hence $\Delta_{c}(\hat{x})>\varepsilon$ and $U_{a(t)}^{b(t)} \subset V_{c, e^{\circ}}$. Consider a set $T$ consisting of only one point $c \in[0,1]$. By virtue of Lemma 1, the set $U_{a(t)}^{b(t)}$ is open in the metric $\rho_{T}$. From the separability of the space $X$ in the metric $\rho_{T}$ it follows that $V_{c, \ell}=\cup_{i=1}^{\infty} U_{a_{i}(t)}^{b_{1}(t)}$, where the functions $a_{i}(t), b_{i}(t)$ can have discontinuities only at the single point $c$. This completes the proof of Lemma 2.

2.5. Let probability measures $\mu_{1}, \ldots, \mu_{n}, \ldots$ and $\mu$ be defined on the Borel sets of the metric space $\Omega$. As usual, we say that $\mu_{n}$ converges weakly to $\mu$ if one of the following three equivalent conditions is satisfied:

(1) For any bounded continuous function $f$ defined on $\Omega$,

$$
\int f d \mu_{n} \rightarrow \int f d \mu \text {. }
$$

(2) For any continuous function $f$ defined on $\Omega$ the distribution of $f$ with respect to the measure $\mu_{n}$ converges to the distribution of $f$ with respect to the measure $\mu$ as $n \rightarrow \infty$.

(3) For any open set $U \subset \Omega, \mu_{n}(U) \rightarrow \mu(U)$ if $\mu(U)=\mu(\bar{U})$; the set $\bar{U}$ is the closure of $U$.

Lemma 3. Let probability measures $\mu_{1}, \ldots, \mu_{n}, \ldots$ and $\mu$ be defined on the Borel sets of the separable metric space $\Omega$ and let $G(\Omega)$ be a Banach algebra with identity of bounded continuous functions defined on $\Omega$. Suppose that for any point $\omega_{0} \in \Omega$ and for any closed set $F \subset \Omega\left(\omega_{0} \notin F\right)$ there exists $g \in G(\Omega)$ such that $g\left(\omega_{0}\right)=0$ and $g(\omega)=1$ for all $\omega \in F$. Suppose finally that for every function $g \in G(\Omega)$ the distribution of $g(\omega)$ with respect to the measure $\mu_{n}$ converges to the distribution of $g(\omega)$ with respect to the measure $\mu$ as $n \rightarrow \infty$. Then $\mu_{n}$ converges weakly to $\mu$.

Proof. Let $U$ be an open set in $\Omega$ and $F=\Omega \backslash U$. For any $\varepsilon>0$ there exist a finite set of points $\omega_{1}, \ldots, \omega_{k} \subset U$ and functions $g_{1}, \ldots, g_{k} \subset G(\Omega)$ such that

(1) $g_{i}\left(\omega_{i}\right)=0$ for every $i=1, \ldots, k ; g_{i}(\omega)=1$ for all $\omega \in F ; 0<g_{i}(\omega)<$ 1 ;

(2) $\mu\left(\cup_{i=1}^{k} L_{i}\right)>\mu(U)-\varepsilon$, where $L_{i}=\left\{\omega \in \Omega: g_{i}(\omega)<\frac{1}{2}\right\}$.

The function $\sum_{i=1}^{k} g_{i}(\omega)=g_{0}(\omega) \in G(\Omega)$ and hence the distribution of $g_{0}(\omega)$ with respect to the measure $\mu_{n}$ converges to the distribution of $g_{0}(\omega)$ with respect to the measure $\mu$ as $n \rightarrow \infty$. There exists a number $p$ such that $k-\frac{1}{2}<p<k$ and $\mu_{n}(L) \rightarrow \mu(L)$, where $L=\left\{\omega \in \Omega: g_{0}(\omega)<p\right\}$. It is evident that $\cup_{i=1}^{k} L_{i} \subset L \subset U$ and hence 


$$
\mu(L)>\mu(U)-\varepsilon
$$

By analogue, there exists an open set $M \subset \Omega \backslash \bar{U}$ such that $\mu(M) \geqslant \mu(\Omega \backslash \bar{U})$ $-\varepsilon$ and $\mu_{n}(M) \rightarrow \mu(M)$. Let us assume that $\mu(U)=\mu(\bar{U})$. In view of the arbitrariness of $\varepsilon>0$, we obtain

$$
\mu_{n}(U) \rightarrow \mu(U) .
$$

This completes the proof of Lemma 3.

REMARK. The Banach algebra $G(\Omega)$ satisfies all the hypotheses of Tychonoff's theorem [4, I, 9], and hence there exists a compactification $K$ of the space $\Omega$ such that the Banach algebra of all bounded continuous functionals of the space $\Omega$, which can be extended continuously on $K$, is $G(\Omega)$. In $[3, \S 5]$ a proof of proposition equivalent to Lemma 3 is given which uses compactifications. The theory of compactifications is used substantially in study of limit theorems for functionals of measurable random processes (see [3]).

2.6. Definition 2. A functional $f$ defined on the set $X$ is said to be completely continuous if there exists an at most countable set $T \subset[0,1]$ such that $f$ is continuous in the metric $\rho_{T}$.

THEOREM 2. Let random processes $\xi_{1}(t), \ldots, \xi_{n}(t), \ldots$ and $\xi(t)$ without discontinuities of the second kind be defined on the interval $[0,1]$. The following properties (A), (B) and (C) are equivalent:

(A) This property consists of two conditions.

I. For any finite set of points $t_{1}, \ldots, t_{k} \subset[0,1]$ the joint distribution of

$$
\xi_{n}\left(t_{1}-0\right), \xi_{n}\left(t_{1}\right), \ldots, \xi_{n}\left(t_{k}-0\right), \xi_{n}\left(t_{k}\right)
$$

converges to the joint distribution of

$$
\xi\left(t_{1}-0\right), \xi\left(t_{1}\right), \ldots, \xi\left(t_{k}-0\right), \xi\left(t_{k}\right) .
$$

II. For any $\varepsilon>0$,

$$
\lim _{c \rightarrow 0} \varlimsup_{n \rightarrow \infty} P\left\{\Delta_{c}\left(\xi_{n}(t)\right)>\varepsilon\right\}=0 .
$$

(B) For any completely continuous functional $f$ the distribution of $f\left(\xi_{n}(t)\right)$ converges to the distribution of $f(\xi(t))$ as $n \rightarrow \infty$.

(C) For any domain $V$,

$$
\lim _{n \rightarrow \infty} P\left\{\xi_{n}(t) \in V\right\}=P\{\xi(t) \in V\}
$$

if

$$
P\{\xi(t) \in V\}=P\{\xi(t) \in \bar{V}\} .
$$

Proof. (1). (A) implies (B). There exists an at most countable set $R_{n} \subset$ $[0,1]$ such that $t \in R_{n}$ if and only if $P\left\{\left|\xi_{n}(t-0)-\xi_{n}(t)\right|>0\right\}>0$. Analogously, there exists an at most countable set $R_{0} \subset[0,1]$ such that 
$t \in R_{0}$ if and only if $P\{|\xi(t-0)-\xi(t)|>0\}>0$. The set $R=\cup_{n=0}^{\infty} R_{n}$. Let an at most countable set $T \subset[0,1] \backslash R$. Consider a set $X_{T} \subset X$. A function $x(t) \in X_{T}$ if and only if $x(t)$ is continuous at all points in $T$. It is evident that

$$
\mu_{1}\left(X_{T}\right)=\cdots=\mu_{n}\left(X_{T}\right)=\cdots=\mu\left(X_{T}\right)=1,
$$

where $\mu_{n}$ is the probability measure corresponding to the process $\xi_{n}(t)$, and $\mu$ is the probability measure corresponding to the process $\xi(t)$. On $X_{T}$ the topology defined by $\rho_{T}$ coincides with the topology defined by the Skorohod metric. This means that for any set $U \subset X$ which is open in the metric $\rho_{T}$ there eixsts a set $U^{\prime} \subset X$ which is open in the Skorohod metric such that

$$
U \cap X_{T} \equiv U^{\prime} \cap X_{T} \text { and }[U] \cap X_{T} \equiv \bar{U}^{\prime} \cap X_{T},
$$

where $[U]$ is the closure of $U$ in the metric $\rho_{T}$, and $\bar{U}^{\prime}$ is the closure of $U^{\prime}$ in the Skorohod metric. Consider a set $U$ which is open in the metric $\rho_{T}$ such that $\mu(U)=\mu([U])$. It follows from (1) that $\mu_{n}\left(U^{\prime}\right)=\mu_{n}(U), \mu\left(U^{\prime}\right)=\mu(U)$ $=\mu([U])=\mu\left(\bar{U}^{\prime}\right)$. By virtue of Theorem 1 ,

$$
\mu_{n}\left(U^{\prime}\right) \rightarrow \mu\left(U^{\prime}\right)
$$

because of the fact that $\mu\left(U^{\prime}\right)=\mu\left(\bar{U}^{\prime}\right)$. Hence

$$
\mu_{n}(U) \rightarrow \mu(U) \text {. }
$$

We have proven that for any functional $f$ which is continuous in the metric $\rho_{T}$ $(T \subset[0,1] \backslash R)$ the distribution of $f\left(\xi_{n}(t)\right)$ converges to the distribution of $f(\xi(t))$ as $n \rightarrow \infty$. From this it follows that if $g(x)$ is a functional which is continuous in the Skorohod metric and $t_{1}, \ldots, t_{k} \subset[0,1] \backslash R$ then the joint distribution of

$$
\xi_{n}\left(t_{1}\right), \ldots, \xi_{n}\left(t_{k}\right), g\left(\xi_{n}(t)\right)
$$

converges to the joint distribution of

$$
\xi\left(t_{1}\right), \ldots, \xi\left(t_{k}\right), g(\xi(t))
$$

as $n \rightarrow \infty$. Consider now an arbitrary finite set of points $t_{1}, \ldots, t_{k} \subset[0,1]$. Let $t_{i}^{\prime} \leqslant t_{i}<t_{i}^{\prime \prime}$, where $t_{i}^{\prime}, t_{i}^{\prime \prime} \subset[0,1] \backslash R(i=1, \ldots, k)$. We have proven that the joint distribution of

$$
\xi_{n}\left(t_{1}^{\prime}\right), \xi_{n}\left(t_{1}^{\prime \prime}\right), \ldots, \xi_{n}\left(t_{k}^{\prime}\right), \xi_{n}\left(t_{k}^{\prime \prime}\right), g\left(\xi_{n}(t)\right)
$$

converges to the joint distribution of

$$
\xi\left(t_{1}^{\prime}\right), \xi\left(t_{1}^{\prime \prime}\right), \ldots, \xi\left(t_{k}^{\prime}\right), \xi\left(t_{k}^{\prime \prime}\right), g(\xi(t))
$$

as $n \rightarrow \infty$. From condition I it follows that the joint distributions of

$$
\xi_{n}\left(t_{i}^{\prime}\right), \xi_{n}\left(t_{i}-0\right) \text { and } \xi_{n}\left(t_{i}\right), \xi_{n}\left(t_{i}^{\prime \prime}\right)
$$

converge, correspondingly, to the joint distributions of 


$$
\xi\left(t_{i}^{\prime}\right), \xi\left(t_{i}-0\right) \text { and } \xi\left(t_{i}\right), \xi\left(t_{i}^{\prime \prime}\right)
$$

as $n \rightarrow \infty$. Now we can easily prove the following proposition: For any finite set of points $t_{1}, \ldots, t_{k} \subset[0,1]$ and for any functional $g(x)$ which is continuous in the Skorohod metric the joint distribution of

$$
\xi_{n}\left(t_{1}-0\right), \xi_{n}\left(t_{1}\right), \ldots, \xi_{n}\left(t_{k}-0\right), \xi_{n}\left(t_{k}\right), g\left(\xi_{n}(t)\right)
$$

converges to the joint distribution of

$$
\xi\left(t_{1}-0\right), \xi\left(t_{1}\right), \ldots, \xi\left(t_{k}-0\right), \xi\left(t_{k}\right), g(\xi(t))
$$

as $n \rightarrow \infty$. Let $\hat{f}$ be a completely continuous functional. There exists an at most countable set $\hat{T} \subset[0,1]$ such that $\hat{f}$ is continuous in the metric $\rho_{\dot{T}}$. Consider functionals

$$
\begin{aligned}
h^{-}(x) & =\max \left[-\alpha, \min \left\{\alpha, f_{t_{0}}^{-}(x)\right\}\right], \quad h(x)=\max \left[-\alpha, \min \left\{\alpha, f_{t_{0}}(x)\right\}\right], \\
\alpha & >0, f_{t_{0}}^{-}(x)=x\left(t_{0}-0\right), \quad f_{t_{0}}(x)=x\left(t_{0}\right), t_{0} \in \hat{T} .
\end{aligned}
$$

Let $Q(X)$ denote a Banach algebra with identity of functionals which is generated by all bounded functionals which are continuous in the Skorohod metric, and all functionals of the form $h^{-}(x), h(x)$. For any function $x_{0} \in X$ and any set $F \subset X\left(x_{0} \notin F\right)$ which is closed in the metric $\rho_{\dot{T}}$ there exists a functional $q(x) \in Q(X)$ such that $q\left(x_{0}\right)=0$ and $q(x)=1$ for $x \in F$. From the above it follows that for any functional $q(x) \in Q(X)$ the distribution of $q\left(\xi_{n}(t)\right)$ converges to the distribution of $q(\xi(t))$ as $n \rightarrow \infty$. From Lemma 3 it follows that the distribution of $\hat{f}\left(\xi_{n}(t)\right)$ converges to the distribution of $\hat{f}(\xi(t))$ as $n \rightarrow \infty$. So the fact that property (A) implies property (B) has been proven.

(2). (B) implies (C). Consider a domain $V=\cup_{i=1}^{\infty} U_{a_{i}(t)}^{b_{1}(t)}$. Let us assume that $\mu(V)=\mu(\bar{V})$. Let $T$ denote an at most countable set on $[0,1]$ such that all the points of discontinuity of the functions $a_{i}(t)$ and $b_{i}(t)$ belong to $T$. Let [V] denote the closure of $V$ in the metric $\rho_{T}$. It is evident that $V \subset[V] \subset \bar{V}$ and hence $\mu(V)=\mu([V])$. The fact that $V$ is a set which is open in the metric $\rho_{T}$ implies immediately that $\mu_{n}(V) \rightarrow \mu(V)$. So we have shown that property (B) implies property (C).

(3). (C) implies (A). Choose a finite number of points $T=\left\{t_{1}<t_{2}\right.$ $\left.<\cdots<t_{k}\right\}$ on the interval $[0,1]$. Consider

$$
\begin{aligned}
W=\left\{x(t): \alpha_{1}^{\prime}<x\left(t_{1}-0\right)\right. & <\beta_{1}^{\prime}, \alpha_{1}^{\prime \prime}<x\left(t_{1}\right)<\beta_{1}^{\prime \prime}, \ldots, \alpha_{k}^{\prime} \\
& \left.<x\left(t_{k}-0\right)<\beta_{k}^{\prime}, \alpha_{k}^{\prime \prime}<x\left(t_{k}\right)<\beta_{k}^{\prime \prime}\right\}, \\
{[W]=\left\{x(t): \alpha_{1}^{\prime}<x\left(t_{1}-0\right)\right.} & <\beta_{1}^{\prime}, \alpha_{1}^{\prime \prime}<x\left(t_{1}\right)<\beta_{1}^{\prime \prime}, \ldots, \alpha_{k}^{\prime} \\
& \left.<x\left(t_{k}-0\right)<\beta_{k}^{\prime}, \alpha_{k}^{\prime \prime}<x\left(t_{k}\right)<\beta_{k}^{\prime \prime}\right\},
\end{aligned}
$$

where $\alpha_{1}^{\prime}<\beta_{1}^{\prime}, \alpha_{1}^{\prime \prime}<\beta_{1}^{\prime \prime}, \ldots, \alpha_{k}^{\prime}<\beta_{k}^{\prime}, \alpha_{k}^{\prime \prime}<\beta_{k}^{\prime \prime}$ is an arbitrary set of real numbers. For any function $x(t) \in W$ there exists a set $U_{a(t)}^{b(t)}$ such that 
$x(t) \in U_{a(t)}^{b(t)} \subset W$ and the functions $a(t), b(t)$ can have discontinuities only at points of the set $T$. By virtue of Lemma 1 the set $U_{a(t)}^{b(t)}$ is open in the metric $\rho_{T}$. The space $X$ is separable in the metric $\rho_{T}$. Hence $W$ is a domain. It can be proven analogously that $X \backslash[W]$ is a domain. Let us assume that $\mu(W)=$ $\mu([W])$. For any $\varepsilon>0$ there exist two finite collections of sets

$$
U_{a_{1}(t)}^{b_{1}(t)}, \ldots, U_{a_{m}(t)}^{b_{m(t)}} ; \quad U_{a^{1}(t)}^{b^{1}(t)}, \ldots, U_{a^{m}(t)}^{b^{m}(t)}
$$

such that

(a) $U_{a_{i}(t)}^{b_{(}(t)} \subset W, \quad U_{a^{\prime}(t)}^{b^{\prime}(t)} \subset X \backslash[W]$ for all $i=1, \ldots, m$;

(b) $\mu\left(U_{a_{i}(t)}^{b_{1}(t)}\right)=\mu\left(\bar{U}_{a_{i}(t)}^{b_{i}(t)}\right), \quad \mu\left(U_{a^{i}(t)}^{b^{i}(t)}\right)=\mu\left(\bar{U}_{a^{i}(t)}^{b^{i}(t)}\right)$;

$$
\text { (c) } \mu\left(\bigcup_{i=1}^{m}\left(U_{a_{i}(t)}^{b_{i}(t)} \cup U_{a^{i}(t)}^{b^{i}(t)}\right)\right)>1-\varepsilon \text {. }
$$

Consider domains $V=\bigcup_{i=1}^{m} U_{a_{i}(t)}^{b_{i}(t)}$ and $V^{\prime}=\bigcup_{i=1}^{m} U_{a^{\prime}(t)}^{b^{\prime}(t)}$. It is evident that $\mu(V)=\mu(\bar{V}), \mu\left(V^{\prime}\right)=\mu\left(\bar{V}^{\prime}\right)$. From (2) and in view of the arbitrariness of $\varepsilon>0$ we obtain

$$
\mu_{n}(W) \rightarrow \mu(W) .
$$

So condition I holds true. Let us prove now that condition II holds true.

Lemma 2 asserts that $V_{c, e}$ is a domain. It can easily be seen that the closure of the domain $V_{c, e}$

$$
\bar{V}_{c, \varepsilon} \subset \bigcap_{p>0} V_{c+p, \varepsilon-p}
$$

From the fact that for $p>0$ the function $\psi(p)=\mu\left(V_{c+p, \varepsilon-p}\right)$ is nondecreasing it follows that there exists an arbitrarily small $p^{\prime}>0$ such that

$$
\mu\left(V_{c+p^{\prime}, \varepsilon-p^{\prime}}\right)=\mu\left(\bar{V}_{c+p^{\prime}, \varepsilon-p^{\prime}}\right) .
$$

Hence

$$
\lim _{n \rightarrow \infty} P\left\{\Delta_{c+p^{\prime}}\left(\xi_{n}(t)\right)>\varepsilon-p^{\prime}\right\}=P\left\{\Delta_{c+p^{\prime}}(\xi(t))>\varepsilon-p^{\prime}\right\} .
$$

So we have proven that condition II holds true. The proof of Theorem 2 is complete.

2.7. Consider random processes $\xi_{1}(t), \ldots, \xi_{n}(t), \ldots$ and $\xi(t)$ on $[0,1]$, without discontinuities of the second kind. These random processes correspond, on $X$, to probability measures $\mu_{1}, \ldots, \mu_{n}, \ldots$ and $\mu$. For every positive number $c<1$ define the subset of $X$

$$
X_{c}=\{x(t): x(c-0)=x(c)\} .
$$

Besides an at most countable set of numbers $c$

$$
\mu_{1}\left(X_{c}\right)=\cdots=\mu_{n}\left(X_{c}\right)=\cdots=\mu\left(X_{c}\right)=1 .
$$

Lemma 2 asserts that the set $V_{c, \varepsilon}=\cup_{i=1}^{\infty} U_{a_{i}(t)}^{b_{i}(t)}$ where the functions $a_{i}(t), b_{i}(t)$ 
can have discontinuities only at the point $c$. From the proof of Lemma 2 it follows that if for $c$, (3) holds, then there exists a domain $V=\cup_{i=1}^{\infty} U_{\dot{a}_{i}(t)}^{\dot{b}_{(}(t)}$ such that $V \subset V_{c, \varepsilon}$, all functions $\hat{a}_{i}(t), \hat{b}_{i}(t)$ are continuous and

$$
\mu_{1}\left(V_{c, e} \backslash V\right)=\cdots=\mu_{n}\left(V_{c, e} \backslash V\right)=\cdots=\mu\left(V_{c, e} \backslash V\right)=0 .
$$

From this and from the part of the proof of Theorem 2 where it is proved that (C) implies II, it is easy to obtain the following proposition:

If for every domain $V=\cup_{i=1}^{\infty} U_{a_{i}(t)}^{b_{i}(t)}$ (all functions $a_{i}(t)$ and $b_{i}(t)$ are continuous) $\mu_{n}(V) \rightarrow \mu(V)$ under the condition that $\mu(V)=\mu(\bar{V})$, then for every $\varepsilon>0$

$$
\lim _{c \rightarrow 0} \varlimsup_{n \rightarrow \infty} P\left\{\Delta_{c}\left(\xi_{n}(t)\right)>\varepsilon\right\}=0 .
$$

From the above and from Theorem 1 we immediately obtain the following

THEOREM 3. Let random processes $\xi_{1}(t), \ldots, \xi_{n}(t), \ldots$ and $\xi(t)$ be given on $[0,1]$, without discontinuities of the second kind. Suppose that for every finite set of points $t_{1}, \ldots, t_{k} \subset[0,1]$ the joint distribution of $\xi_{n}\left(t_{1}\right), \ldots, \xi_{n}\left(t_{k}\right)$ converges to the joint distribution of $\xi\left(t_{1}\right), \ldots, \xi\left(t_{k}\right)$ as $n \rightarrow \infty$. Then the following properties $(\mathrm{A})^{\prime},(\mathrm{B})^{\prime},(\mathrm{C})^{\prime}$ are equivalent:

(A)' For every $\varepsilon>0$,

$$
\lim _{c \rightarrow 0} \varlimsup_{n \rightarrow \infty} P\left\{\Delta_{c}\left(\xi_{n}(t)\right)>\dot{\varepsilon}\right\}=0 .
$$

(B)' For every functional $f$ which is continuous in the Skorohod metric the distribution of $f\left(\xi_{n}(t)\right)$ converges to the distribution of $f(\xi(t))$ as $n \rightarrow \infty$.

(C)' For every domain $V=\cup_{i=1}^{\infty} U_{a_{i}(t)}^{b_{i}(t)}$ where $a_{i}(t), b_{i}(t)$ are continuous functions and

$$
P\{\xi(t) \in V\}=P\{\xi(t) \in \bar{V}\}
$$

the equality

$$
\lim _{n \rightarrow \infty} P\left\{\xi_{n}(t) \in V\right\}=P\{\xi(t) \in V\}
$$

holds.

\section{Convergence of random processes with independent increments.}

3.1. Consider a finite set of independent random values $\eta_{1}, \ldots, \eta_{n}$. Let $\Sigma_{k}$ denote the sum $\eta_{1}+\cdots+\eta_{k}$.

LEMMA 4. Let $P\left\{\left|\Sigma_{n}-\Sigma_{k}\right| \geqslant a\right\} \leqslant \alpha, k=1, \ldots, n$, for some positive number $\alpha<1$. Then

$$
P\left\{\sup _{k}\left|\Sigma_{k}\right| \geqslant a+x\right\} \leqslant \frac{1}{1-\alpha} P\left\{\left|\Sigma_{n}\right| \geqslant x\right\} .
$$

Proof. [1, §3]. 
LEMMA 5. If for some positive $\varepsilon>0$ we have

$$
P\left\{\left|\Sigma_{n}-\Sigma_{k}\right|>\frac{\varepsilon}{8}\right\}<\alpha<1, \quad k=1, \ldots, n,
$$

then

$$
P\left\{\sup _{1<i<k<j<n} \min \left[\left|\Sigma_{i}-\Sigma_{k}\right| ;\left|\Sigma_{k}-\Sigma_{j}\right|\right]>\varepsilon\right\} \leqslant \frac{P\left\{\left|\Sigma_{n}\right|>\varepsilon / 8\right\}^{2}}{(1-\alpha)^{2}}
$$

Proof. [1, §40].

3.2. Let $\xi(t)$ be a random process on $[0,1]$. Let two finite set of points $S_{R}=\left(s_{0}, s_{1}, \ldots, s_{r}, \ldots, s_{R}\right)$ and $H_{Q}=\left(h_{1}, \ldots, h_{q}, \ldots, h_{Q}\right)$ be given on $[0,1]$ and let $0=s_{0}<s_{1}<\cdots<s_{R-1}<s_{R}=1, h_{1}<h_{2}<\cdots<h_{Q}$. Let numbers $c>0, \varepsilon>0,0<\alpha<1,0<\beta<1$ be given. We say that $\xi(t), S_{R}, H_{Q}, c, \varepsilon, \alpha, \beta$ is a consistent system if

(1) $s_{r} \neq h_{q}$ for all $r$ and $q$;

(2) $2 c<s_{r}-s_{r-1} \leqslant 4 c$;

(3) $h_{q+1}-h_{q}>8 c, h_{1}>4 c, h_{Q}<1-4 c$;

(4) for every pair of points $t^{\prime}<t^{\prime \prime}$

$$
P\left\{\left|\xi\left(t^{\prime}\right)-\xi\left(t^{\prime \prime}\right)\right|>\varepsilon / 16\right\}<\alpha
$$

if $t^{\prime \prime}-t^{\prime} \leqslant 8 c$ and if there exist no $q$ such that $t^{\prime} \leqslant h_{q} \leqslant t^{\prime \prime}$;

(5) for every pair of points $\hat{t}<\hat{t}$ we have

$$
P\{|\xi(\hat{t})-\xi(\hat{\hat{t}})|>\varepsilon / 32\}<\beta
$$

if there exists a $q$ such that either $-8 c \leqslant \hat{t}<\hat{i}<h_{q}$ or $h_{q} \leqslant \hat{t}<\hat{i}<h_{q}+$ $8 c$.

LEMMA 6. Let $\xi(t)$ be a random process with independent increments on $[0,1]$ which has no discontinuities of the second kind and let $\xi(t), S_{R}, H_{Q}, c, \varepsilon, \alpha, \beta$ be a consistent system. Then

$$
P\left\{\Delta_{c}(\xi(t))>\varepsilon\right\} \leqslant \frac{2 \alpha}{(1-\alpha)^{2}}\left[\sum_{r=1}^{R} P\left\{\left|\xi\left(s_{r}\right)-\xi\left(s_{r-1}\right)\right|>\frac{\varepsilon}{32}\right\}+1\right]+\frac{4 \beta \cdot Q}{1-\beta}
$$

Proof. Let $0=\tau_{0}^{(p)}<\tau_{1}^{(p)}<\cdots<\tau_{m p}^{(p)}=1$ and

$$
\lim _{p \rightarrow \infty} \max _{j}\left(\tau_{j}^{(p)}-\tau_{j-1}^{(p)}\right)=0 \text {. }
$$

Then 
$\Delta_{c}(\xi(t))$

$$
\begin{aligned}
& =\lim _{p \rightarrow \infty}\left\{\sup _{\tau_{j}^{(p)}<c}\left|\xi\left(\tau_{j}^{(p)}\right)-\xi(0)\right|+\sup _{1-\tau_{j}^{(p)}<c}\left|\xi(1)-\xi\left(\tau_{j}^{(p)}\right)\right|\right. \\
& +\sup _{\tau_{j}^{(p)}-c<\tau_{i}^{(p)}<\tau_{i}^{(p)}<\tau_{k}^{(p)}<\tau_{j}^{(p)}+c} \min \left[\left|\xi\left(\tau_{i}^{(p)}\right)-\xi\left(\tau^{(p)}\right)\right| ;\right. \\
& \left.\left.\left|\xi\left(\tau^{(p)}\right)-\xi\left(\tau_{k}^{(p)}\right)\right|\right]\right\} .
\end{aligned}
$$

Let all points $s_{r}$ and $h_{q}$ belong to the set of points $\tau_{j}^{(p)}$ for every $p$. We have

$$
\begin{aligned}
P\left\{\Delta_{c}(\xi(t))>\varepsilon\right\}<\varlimsup_{p \rightarrow \infty} P\left\{\sup _{\tau_{j}^{(p)}<c}\left|\xi\left(\tau^{(p)}\right)-\xi(0)\right|>\frac{\varepsilon}{4}\right\} \\
+\lim _{p \rightarrow \infty} P\left\{\sup _{1-\tau^{(p)}<c}\left|\xi(1)-\xi\left(\tau^{(p)}\right)\right|>\frac{\varepsilon}{4}\right\} \\
+\varlimsup_{p \rightarrow \infty} \sum_{r=0}^{R-2} P\left\{\operatorname { s u p } _ { s _ { r } < \tau _ { i } ^ { ( p ) } < \tau _ { j } ^ { ( p ) } < \tau _ { r } ^ { ( p ) } < s _ { r + 2 } } \operatorname { m i n } \left[\left|\xi\left(\tau_{i}^{(p)}\right)-\xi\left(\tau_{j}^{(p)}\right)\right| ;\right.\right. \\
\left.\left.\left|\xi\left(\tau_{j}^{(p)}\right)-\xi\left(\tau_{k}^{(p)}\right)\right|\right]>\frac{\varepsilon}{2}\right\} .
\end{aligned}
$$

Denote by $S_{r q}$ the point in $S_{R}$ for which $h_{q} \in\left[s_{r^{q}}, s_{r^{q}+1}\right]$ by virtue of Lemmas 4 and 5.

$$
\begin{aligned}
& P\left\{\Delta_{c}(\xi(t))>\varepsilon\right\}<\frac{2 \alpha}{1-\alpha}+\frac{\alpha}{(1-\alpha)^{2}} \sum_{r=0}^{R-2} P\left\{\left|\xi\left(s_{r+2}\right)-\xi\left(s_{r}\right)\right|>\frac{\varepsilon}{16}\right\} \\
& +\varlimsup_{p \rightarrow \infty} \sum_{q=1}^{Q}\left[P \left\{\operatorname { s u p } _ { s _ { r + - 1 } < \tau _ { i } ^ { ( p ) } < \tau _ { j } ^ { ( p ) } < \tau _ { k } ^ { ( p ) } < s _ { r + 1 } } \operatorname { m i n } \left[\left|\xi\left(\tau_{i}^{(p)}\right)-\xi\left(\tau_{j}^{(p)}\right)\right| ;\right.\right.\right. \\
& +P\left\{\sup _{s_{r}<\tau_{i}^{(p)}<\tau_{j}^{(p)}<\tau_{k}^{(p)}<s_{r+2}} \min \left[\left|\xi\left(\tau_{i}^{(p)}\right)-\xi\left(\tau_{k}^{(p)}\right)\right|\right]>\frac{\varepsilon}{2}\right\} \\
&
\end{aligned}
$$




$$
\begin{aligned}
& <\frac{2 \alpha}{(1-\alpha)^{2}}\left[\sum_{r=1}^{R} P\left\{\left|\xi\left(s_{r}\right)-\xi\left(s_{r-1}\right)\right|>\frac{\varepsilon}{32}\right\}+1\right] \\
& +2 \varlimsup_{p \rightarrow \infty} \sum_{q=1}^{Q}\left[P\left\{\sup _{\substack{s_{p-1}<\tau_{i}^{(p)}<\tau_{j}^{(p)}<h_{q}\\
}}\left|\xi\left(\tau_{i}^{(p)}\right)-\xi\left(\tau_{j}^{(p)}\right)\right|>\frac{\varepsilon}{4}\right\}\right. \\
& \left.+P\left\{\sup _{h_{q}<\tau_{j}^{(p)}<\tau_{k}^{(p)}<s_{, q+2}}\left|\xi\left(\tau_{j}^{(p)}\right)-\xi\left(\tau_{k}^{(p)}\right)\right|>\frac{\varepsilon}{4}\right\}\right] \\
& <\frac{2 \alpha}{(1-\alpha)^{2}}\left[\sum_{r=1}^{R} P\left\{\left|\xi\left(s_{r}\right)-\xi\left(s_{r-1}\right)\right|>\frac{\varepsilon}{32}\right\}+1\right] \\
& +2 \varlimsup_{p \rightarrow \infty} \sum_{q=1}^{Q}\left[P\left\{\sup _{s_{r_{q}-1}<\tau_{j}^{(p)}<h_{q}}\left|\xi\left(s_{r^{q}-1}\right)-\xi\left(\tau_{j}^{(p)}\right)\right|>\frac{\varepsilon}{8}\right\}\right. \\
& \left.+P\left\{\sup _{h_{q}<\tau_{j}^{(p)}<s_{p q+2}}\left|\xi\left(s^{q}\right)-\xi(\tau(p))\right|>\frac{\varepsilon}{8}\right\}\right] \\
& <\frac{2 \alpha}{(1-\alpha)^{2}}\left[\sum_{r=1}^{R} P\left\{\left|\xi\left(s_{r}\right)-\xi\left(s_{r-1}\right)\right|>\frac{\varepsilon}{32}\right\}+1\right]+\frac{4 \beta \cdot Q}{1-\beta}
\end{aligned}
$$

and Lemma 6 is proved.

REMARK. The proof of Lemma 6 is analogous to the proof of Lemma 2 in $[1, \S 40]$. The latter is a particular case of Lemma 6 under the assumption that the set $H_{Q}$ is empty.

3.3. Here we shall prove Theorem 4 which was formulated in the Introduction.

Necessity. Condition (a) is condition I of Theorem 2. Its necessity follows from Theorem 2.

For the proof of necessity of condition (b) we point out, first of all, that there exists an $A$ such that

$$
E \xi_{n}^{2}(t) \leqslant A, \quad E \xi_{n}^{2}(t-0) \leqslant A, \quad E \xi^{2}(t) \leqslant A, \quad E \xi^{2}(t-0) \leqslant A
$$

for all $n$ and $t$. The function $\varphi(t)=E \xi(t)$ and the functions $\varphi_{n}(t)$ have no discontinuities of the second kind, and for all $t$ we have

$$
\varphi_{n}(t) \rightarrow \varphi(t), \quad \varphi_{n}(t-0) \rightarrow \varphi(t-0) .
$$

One must prove that $\varphi_{n}(t)$ converges uniformly to $\varphi(t)$. For $\varepsilon>0$ let us fix a finite number of points $0=t_{0}<t_{1}<\cdots<t_{k}<t_{k+1}=1$ such that if $t \neq t_{i}$, where $i=1, \ldots, k, E|\xi(t-0)-\xi(t)|<\varepsilon$. There exists $\delta>0$ such that if $t^{\prime}, t^{\prime \prime} \subset\left[t_{i-1}, t_{i}\right)$ or $t^{\prime}, t^{\prime \prime} \subset\left[t_{k}, 1\right]$ and $\left|t^{\prime}-t^{\prime \prime}\right|<\delta$ then $E \mid \xi\left(t^{\prime}\right)-$ 
$\xi\left(t^{\prime \prime}\right) \mid<\varepsilon$. Let $\sigma=\min _{1<i<k+1}\left(t_{i}-t_{i-1}\right)$. Consider a positive number $c<$ $\min (\sigma / 2, \delta / 2)$. There exists a natural number $N_{1}$ such that:

(1) for $n \geqslant N_{1}$ we have $\left|\varphi_{n}\left(t_{i}-0\right)-\varphi\left(t_{i}-0\right)\right|<\varepsilon,\left|\varphi_{n}\left(t_{i}\right)-\varphi\left(t_{i}\right)\right|<\varepsilon$ for all $i=0,1, \ldots, k, k+1$;

(2) for $n>N_{1}$ and for every $i=1, \ldots, k$ there exist two points $\tau_{i, 1}^{(n)}, \tau_{i, 2}^{(n)}$ such that

$$
\begin{gathered}
\tau_{i, 1}^{(n)} \in\left[t_{i}-2 c, t_{i}-c\right], \quad \tau_{i, 2}^{(n)} \in\left[t_{i}+c, t_{i}+2 c\right], \\
|E| \xi_{n}\left(t_{i}-0\right)-\xi_{n}\left(\tau_{i, 1}^{(n)}\right)|-E| \xi\left(t_{i}-0\right)-\xi\left(\tau_{i, 1}^{(n)}\right)||<\varepsilon, \\
|E| \xi_{n}\left(t_{i}\right)-\xi_{n}\left(\tau_{i, 2}^{(n)}\right)|-E| \xi\left(t_{i}\right)-\xi\left(\tau_{i, 2}^{(n)}\right)||<\varepsilon, \\
\left|\varphi_{n}\left(\tau_{i, 1}^{(n)}\right)-\varphi\left(\tau_{i, 1}^{(n)}\right)\right|<\varepsilon, \quad\left|\varphi_{n}\left(\tau_{i, 2}^{(n)}\right)-\varphi\left(\tau_{i, 2}^{(n)}\right)\right|<\varepsilon ;
\end{gathered}
$$

(3) for $n \geqslant N_{1}$ and for every point $t \in[c, 1-c]$ there exist two points $t_{n}^{1}, t_{n}^{2}$ such that

$$
\begin{gathered}
t-c<t_{n}^{1}<t<t_{n}^{2}<t+c, \\
|E| \xi_{n}\left(t_{n}^{1}\right)-\xi_{n}\left(t_{n}^{2}\right)|-E| \xi\left(t_{n}^{1}\right)-\xi\left(t_{n}^{2}\right)||<\varepsilon, \\
\left|\varphi_{n}\left(t_{n}^{1}\right)-\varphi\left(t_{n}^{1}\right)\right|<\varepsilon, \\
\left|\varphi_{n}\left(t_{n}^{2}\right)-\varphi\left(t_{n}^{2}\right)\right|<\varepsilon .
\end{gathered}
$$

Suppose that there exist $\hat{t}$ and $n_{0}>N_{1}$ such that

$$
\left|\varphi_{n_{0}}(\hat{t})-\varphi(\hat{t})\right| \geqslant 4 \varepsilon
$$

We have three possibilities:

(1) $\hat{t} \notin(c, 1-c)$;

(2) there exists $i=1, \ldots, k$ such that $\left|t_{i}-\hat{t}\right|<\varepsilon$;

(3) there exists $i=0,1, \ldots, k$ such that $t_{i}+c<\hat{i}<t_{i+1}-c$.

Case (1). Let $\hat{t} \in(0, c]$. Then

$$
E\left|\xi_{n_{0}}(0)-\xi_{n_{0}}(\hat{t})\right|>\left|\varphi_{n_{0}}(0)-\varphi_{n_{0}}(\hat{t})\right|>2 \varepsilon .
$$

Consider a number $q_{1}>\max (1, \varepsilon, 8 A / \varepsilon)$. It can easily be shown that

$$
P\left\{\left|\xi_{n_{0}}(0)-\xi_{n_{0}}(\hat{t})\right|>\varepsilon\right\}>\varepsilon / 2 q_{1} .
$$

Therefore if $t \notin(c, 1-c)$ then

$$
P\left\{\Delta_{c}\left(\xi_{n_{0}}(t)\right)>\varepsilon\right\}>\varepsilon / 2 q_{1} .
$$

Case (2). Let $0<\hat{i}-t_{i}<c, 1<i<k$. Consider a random value

$$
\eta=\min \left\{\left|\xi_{n_{0}}\left(t_{i}\right)-\xi_{n_{0}}(\hat{t})\right| ;\left|\xi_{n_{0}}(\hat{t})-\xi_{n_{0}}\left(\tau_{i, 2}^{\left(n_{0}\right)}\right)\right|\right\} .
$$

It can easily be seen that 


$$
\eta \geqslant \frac{\left|\xi_{n_{0}}\left(t_{i}\right)-\xi_{n_{0}}(\hat{t})\right|+\left|\xi_{n_{0}}(\hat{t})-\xi_{n_{0}}\left(\tau_{i, 2}^{\left(n_{0}\right)}\right)\right|}{2}-\frac{\left|\xi_{n_{0}}\left(t_{i}\right)-\xi_{n_{0}}\left(\tau_{i, 2}^{\left(n_{0}\right)}\right)\right|}{2}
$$

and therefore

$$
E \eta \geqslant \frac{\left|\varphi_{n_{0}}\left(t_{i}\right)-\varphi_{n_{0}}(\hat{t})\right|+\left|\varphi_{n_{0}}(\hat{t})-\varphi_{n_{0}}\left(\tau_{i, 2}^{\left(n_{0}\right)}\right)\right|}{2}-\varepsilon>\varepsilon .
$$

Consider a number $q_{2}>\max (1, \varepsilon / 2 ; 16 A / \varepsilon)$. It is easy to prove that

$$
P\{\eta>\varepsilon / 2\} \geqslant \varepsilon / 4 q_{2} \text {. }
$$

Therefore if $\left|t_{i}-\hat{t}\right| \leqslant c(1 \leqslant i \leqslant k)$ then

$$
P\left\{\Delta_{2 c}\left(\xi_{n_{0}}(t)\right)>\varepsilon / 2\right\}>\varepsilon / 4 q_{2} .
$$

Case (3). Let $t_{i}+c<\hat{t}<t_{i+1}-c$. Let us choose points $t_{n_{n^{\prime}}}^{1}, t_{n_{0}}^{2}$ such that $t_{i}<\hat{t}-c<t_{n_{0}}^{1}<\hat{t}<t_{n_{0}}^{2}<\hat{t}+c<t_{i+1}$ and conditions (4), (5), (6) are satisfied for $n=n_{0}$. Consider a random value

$$
\chi=\min \left\{\left|\xi_{n_{0}}\left(t_{n_{0}}^{1}\right)-\xi_{n_{0}}(\hat{t})\right| ;\left|\xi_{n_{0}}(\hat{t})-\xi_{n_{0}}\left(t_{n_{0}}^{2}\right)\right|\right\} .
$$

It can be proven as in case (2) that $P\{\chi>\varepsilon / 2\}>\varepsilon / 4 q_{2}$. Therefore if there exists $i=0,1, \ldots, k$ such that $t_{i}+c<\hat{i}<t_{i+1}-c$ then

$$
P\left\{\Delta_{c}\left(\xi_{n_{0}}(t)\right)>\varepsilon / 2\right\} \geqslant \varepsilon / 4 q_{2} .
$$

Let us summarize: if there exist $\hat{t}$ and $n_{0} \geqslant N_{1}$ such that $\left|\varphi_{n_{0}}(\hat{t})-\varphi(\hat{t})\right| \geqslant 4 \varepsilon$ then

$$
P\left\{\Delta_{2 c}\left(\xi_{n_{0}}(t)\right)>\frac{\varepsilon}{2}\right\} \geqslant \frac{\varepsilon}{4 q}
$$

where $q=\max \left(q_{1}, q_{2}\right)$. By virtue of Theorem 2 there exist $c_{0}>0$ and a natural number $N_{2}$ such that for $n \geqslant N_{2}$,

$$
P\left\{\Delta_{c_{0}}\left(\xi_{n}(t)\right)>\frac{\varepsilon}{2}\right\}<\frac{\varepsilon}{4 q} .
$$

Set $c<c_{0} / 2$ and $N=\max \left(N_{1}, N_{2}\right)$. For $n \geqslant N$,

$$
\left|\varphi_{n}(t)-\varphi(t)\right|<4 \varepsilon .
$$

The uniform convergence of $\varphi_{n}(t)$ to $\varphi(t)$ is proved.

Sufficiency. Suppose, without loss of generality, that $E \xi_{n}(t)=0$ and $E \xi(t)$ $=0$ for all $n$ and $t$. Let us fix numbers $\varepsilon>0$ and $0<\alpha<1$. There exists an at most finite set of points $H_{Q}=\left(h_{1}<\cdots<h_{q}<\cdots<h_{Q}\right)$ such that

$$
D\left(\xi\left(h_{q}-0\right)-\xi\left(h_{q}\right)\right) \geqslant\left(\alpha \cdot \varepsilon^{2}\right) / 256
$$

We shall prove that there exist a number $c_{1}>0$ and a natural number $N^{1}$ such that

$$
h_{1} \geqslant 4 c_{1}, \quad 1-h_{Q}>4 c_{1}, \quad h_{q+1}-h_{q}>8 c_{1}
$$


and for all pairs of points $t^{\prime}<t^{\prime \prime}$ and for every $n \geqslant N^{\prime}$,

$$
P\left\{\left|\xi_{n}\left(t^{\prime}\right)-\xi_{n}\left(t^{\prime \prime}\right)\right|>\varepsilon / 16\right\}<\alpha
$$

if $t^{\prime \prime}-t^{\prime}<8 c$ and there exists no $q$ such that $t^{\prime} \leqslant h_{q}<t^{\prime \prime}$. Suppose the contrary. This means that there exist point $t_{0}$ and two sequences of points $t_{n_{1}}^{(1)}$ and $t_{n_{j}}^{(2)}, n_{j} \rightarrow \infty, t_{n_{j}}^{(1)} \rightarrow t_{0}, t_{n_{j}}^{(2)} \rightarrow t_{0}$ such that

$$
D\left(\xi_{n_{j}}\left(t_{n_{j}}^{(1)}\right)-\xi_{n_{j}}\left(t_{n_{j}}^{(2)}\right)\right) \geqslant\left(\alpha \cdot \varepsilon^{2}\right) / 256
$$

If $t_{0}=h_{q}$ then $t_{n_{j}}^{(1)}$ and $t_{n_{j}}^{(2)}$ converge to $t_{0}$ simultaneously either from the left or from the right, and $t_{0} \neq t_{n_{j}}^{(1)}, t_{0} \neq t_{n_{j}}^{(2)}$. Let $(0,1) \ni t_{0} \neq h_{q}$. Then there exist two points $t_{1}$ and $t_{2}$ such that $t_{1}<t_{0}<t_{2}$ and

$$
D\left(\xi\left(t_{1}\right)-\xi\left(t_{2}\right)\right)<\left(\alpha \cdot \varepsilon^{2}\right) / 256 .
$$

On the other hand, there exists a number $J$ such that for $j \geqslant J$ we have $t_{n_{j}}^{(1)}, t_{n_{j}}^{(2)} \subset\left[t_{1}, t_{2}\right]$. Therefore for $j \geqslant J$ we have

$$
D\left(\xi_{n_{j}}\left(t_{1}\right)-\xi_{n_{j}}\left(t_{2}\right)\right) \geqslant\left(\alpha \cdot \varepsilon^{2}\right) / 256 .
$$

But $D\left(\xi_{n}\left(t_{1}\right)-\xi_{n}\left(t_{2}\right)\right) \rightarrow D\left(\xi\left(t_{1}\right)-\xi\left(t_{2}\right)\right)<\left(\alpha \cdot \varepsilon^{2}\right) / 256$, a contradiction. If $t_{0}=0$ or 1 or $h_{q}$ then we also arrive at a contradiction.

Let us fix a positive number $\beta<1$. There exists a positive number $c \leqslant c_{1} / 2$ such that for all $q=1, \ldots, Q$ we have

$$
\begin{gathered}
D\left(\xi\left(h_{q}-8 c\right)-\xi\left(h_{q}-0\right)\right)<\left(\beta \cdot \varepsilon^{2}\right) / 1024 \text { and } \\
D\left(\xi\left(h_{q}\right)-\xi\left(h_{q}+8 c\right)\right)<\left(\beta \cdot \varepsilon^{2}\right) / 1024 .
\end{gathered}
$$

There exists a natural number $N^{2} \geqslant N^{1}$ such that for every pair of points $\hat{t}<\hat{\hat{t}}$ we have

$$
P\left\{\left|\xi_{n}(\hat{t})-\xi_{n}(\hat{\hat{t}})\right|>\varepsilon / 32\right\}<\beta
$$

if $n \geqslant N^{2}$ and there exists $q$ such that either $h_{q}-8 c \leqslant \hat{t}<\hat{t}<h_{q}$ or $h_{q}<\hat{t}<\hat{t}<h_{q}+8 c$.

Consider a set of points $S_{R}=\left\{0=s_{0}<s_{1}<\cdots<s_{r}<\cdots<s_{R}=1\right\}$ such that $s_{r} \neq h_{q}$ for all $r$ and $q, 2 c<s_{r}-s_{r-1} \leqslant 4 c$. For all $n>$ $N^{2}, \xi_{n}(t), S_{R}, H_{Q}, c, \varepsilon, \alpha, \beta$ is a consistent system. By virtue of Lemma 6 for $n>N^{2}$ we have

$$
\begin{aligned}
& P\left\{\Delta_{c}\left(\xi_{n}(t)\right)>\varepsilon\right\} \\
& \quad<\frac{2 \alpha}{(1-\alpha)^{2}}\left[\sum_{r=1}^{R} P\left\{\left|\xi_{n}\left(s_{r}\right)-\xi_{n}\left(s_{r-1}\right)\right|>\frac{\varepsilon}{32}\right\}+1\right]+\frac{4 \beta \cdot Q}{1-\beta} \\
& \quad \leqslant \frac{2 \alpha}{(1-\alpha)^{2}}\left[\frac{1024}{\varepsilon^{2}} D\left(\xi_{n}(0)-\xi_{n}(1)\right)+1\right]+\frac{4 \beta \cdot Q}{1-\beta} .
\end{aligned}
$$


Therefore

$$
\varlimsup_{n \rightarrow \infty} P\left\{\Delta_{c}\left(\xi_{n}(t)\right)>\varepsilon\right\} \leqslant \frac{2 \alpha}{(1-\alpha)^{2}}\left[\frac{1024}{\varepsilon^{2}} D(\xi(0)-\xi(1))+1\right]+\frac{4 \beta \cdot Q}{1-\beta} .
$$

From the above it follows that condition II of Theorem 2 for random processes $\xi_{n}(t)$ holds true. By assumption, condition $I$ of Theorem 2 for the processes $\xi_{n}(t)$ and $\xi(t)$ is satisfied. By virtue of the above and Theorem 2 we have the validity of Theorem 4.

REMARK. In the proof of the necessity of conditions (a) and (b) of Theorem 4 the fact that $\xi_{n}(t)$ and $\xi(t)$ are random processes with independent increments was never used.

3.4. Definition 3. A random process $\xi(t)$ without discontinuities of the second kind defined on $[0,1]$ is said to be almost continuous if there exists an at most countable set $T_{\xi} \subset[0,1]$ such that the sample functions of $\xi(t)$ have no discontinuities at the points of $[0,1] \backslash T_{\xi}$ with probability 1 .

Let $\xi(t)$ be an almost continuous process. Consider a set

$$
X^{\prime}=\left\{x \in X: x(t-0)=x(t) \text { if } t \in[0,1] \backslash T_{\xi}\right\} .
$$

It is evident that

$$
P\left\{\xi(t) \in X^{\prime}\right\}=1 \text {. }
$$

Consider the uniform metric $\rho_{c}$ on $X^{\prime}$. The sets of the form $U_{a(t)}^{b(t)}$, where the functions $a(t)$ and $b(t)$ can have discontinuities only at points of $T$, generate the basis of open sets of the space $X^{\prime}$ with the metric $\rho_{c}$. From this it follows that the topologies determined on $X^{\prime}$ by the metric $\rho_{c}$ and $\rho_{T_{\xi}}$ coincide. The following theorem is an immediate corollary of the above and Theorem 4.

THEOREM 5. Let almost continuous random processes with independent increments $\xi_{1}(t), \ldots, \xi_{n}(t), \ldots$ and $\xi(t)$ be defined on $[0,1]$. Suppose that for every $\varepsilon>0$ there exists $d>0$ such that $E \xi_{n}^{(d)}(t)<\varepsilon$ for all $n$ and $t$. Then the distribution of $f\left(\xi_{n}(t)\right)$ converges to the distribution of $f(\xi(t))$ as $n \rightarrow \infty$ for every functional $f$ which is continuous in the uniform metric if and only if conditions (a) and (b) of Theorem 4 are satisfied.

4. Limit theorems for sums of independent random variables. Let $\eta(t)$ be a random process with independent increments, defined on $[0,1]$ and such that $E|\eta(t)|^{2}<A$, for some $A$ and all $t \in[0,1]$. Let $\varphi(t)=E \eta(t), \bar{\eta}(t)=\eta(t)-$ $\varphi(t)$ and $\psi(t)=D \eta(t)$. The process $\eta(t)$ has no discontinuities of the second kind if and only if the function $\varphi(t)$ has no discontinuities of the second kind. We assume that $\eta(t)$ has no discontinuities of the second kind. Denote by $\tau_{1}, \ldots, \tau_{k}, \ldots$, the points of discontinuities of $\psi(t)$. Set

$$
\hat{\eta}\left(t_{1}, t_{2}\right)=\bar{\eta}\left(t_{2}\right)-\bar{\eta}\left(t_{1}\right)-\sum_{\tau_{k} \in\left(t_{1}, t_{2}\right]}\left\{\bar{\eta}\left(\tau_{k}\right)-\bar{\eta}\left(\tau_{k}-0\right)\right\}
$$


where $t_{1}<t_{2}$. The distribution function of the random variable $\hat{\eta}\left(t_{1}, t_{2}\right)$ is infinitely divisible and thus

$$
\ln \left(E e^{i s \hat{\eta}\left(t_{1}, t_{2}\right)}\right)=\int_{-\infty}^{\infty}\left\{e^{i s x}-1-i s x\right\} \frac{1}{x^{2}} d G\left(x ; t_{1}, t_{2}\right)
$$

where $G\left(x ; t_{1}, t_{2}\right)$ is a nondecreasing function, $G\left(-\infty ; t_{1}, t_{2}\right)=0$, $G\left(\infty ; t_{1}, t_{2}\right)=D \hat{\eta}\left(t_{1}, t_{2}\right)$. Denote by $\Phi_{0}$ the distribution of $\bar{\eta}(0)$, and by $\Phi_{k}$ the distribution of the random variable $\bar{\eta}\left(\tau_{k}\right)-\bar{\eta}\left(\tau_{k}-0\right)$.

Consider a double sequence of random variables

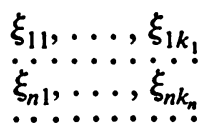

with each row independent. Let us partition the interval $[0,1]$ at the points $0=t_{n 0}<t_{n 1}<\cdots<t_{n k_{n}}=1$, so that $\max _{1 \leqslant k \leqslant k_{n}}\left(t_{n k}-t_{n, k-1}\right) \rightarrow 0$ when $n \rightarrow \infty$. Set

$$
\eta_{n}(t)=\sum_{i=1}^{k} \xi_{n i} \text { if } t_{n, k-1} \leqslant t<t_{n k} \text { and } \eta_{n}(1)=\sum_{i=1}^{k_{n}} \xi_{n i} .
$$

Suppose that for any $\varepsilon>0$ there exists $d>0$ such that $E \eta_{n}^{(d)}(t)<\varepsilon$ for all $n$ and $t$. Denote by $F_{n k}$ the distribution of $\xi_{n k}$ and set $\bar{F}_{n k}=F_{n k}\left(x+E \xi_{n k}\right)$.

THEOREM 6. The distribution $f\left(\eta_{n}(t)\right)$ converges to the distribution $f(\eta(t))$ for any completely continuous functional $f$ if and only if all of the following conditions are satisfied:

(1) The functions $\varphi_{n}(t)=E \eta_{n}(t)$ converge uniformly to $\varphi(t)$;

(2) The functions $\psi_{n}^{\prime}=D \eta_{n}(t)$ converge uniformly to $\psi(t)$;

(3) The distribution of $\eta_{n}(0)$ converges to the distribution of $\eta(0)$;

(4) The distribution of $\eta_{n}\left(\tau_{k}\right)-\eta_{n}\left(\tau_{k}-0\right)$ converges to the distribution of $\eta\left(\tau_{k}\right)-\eta\left(\tau_{k}-0\right)$

(5) For any pair of points $t_{1}<t_{2}$,

$$
\begin{aligned}
\lim _{n \rightarrow \infty} \sum\left\{\int_{-\infty}^{u} x^{2} d \bar{F}_{n k}(x) ; t_{n, k-1} \in\left(t_{1}, t_{2}\right]\right\} \\
=G\left(u ; t_{1}, t_{2}\right)+\sum\left\{\int_{-\infty}^{u} x^{2} d \Phi_{k}(x) ; \tau_{k} \in\left(t_{1}, t_{2}\right]\right\}
\end{aligned}
$$

with the possible exception of a countabie set of numbers $u$.

- By the usual arguments one can prove that the random process is almost continuous if and only if the distributions of the random variables $\hat{\eta}\left(t_{1}, t_{2}\right)$ are normal.

THEOREM 7. Suppose that $\eta(t)$ is an almost continuous process. The distribution of $f\left(\eta_{n}(t)\right)$ converges to the distribution of $f(\eta(t))$ for any functional 
$f$ continuous in the uniform metric, if and only if the conditions (1)-(4) of Theorem 6 are satisfied and

$$
\sum_{k=1}^{k_{n}} \int_{|x|>\delta} x^{2} d \bar{F}_{n k}(x) \rightarrow \sum_{k=0}^{\infty} \int_{|x|>\delta} x^{2} d \Phi_{k}(x)
$$

with the possible exception of a countable set of positive numbers $\delta$.

\section{BIBLIOGRAPHY}

1. A. V. Skorohod, Random processes with independent increments, "Nauka", Moscow, 1964; English transl.. Theory of random processes, National Lending Library for Science and Technology, Boston Spa, Yorkshire, England, 1971. MR 31 \#6280.

2. I. I. Gihman and A. V. Skorohod, The theory of stochastic processes. I, "Nauka", Moscow, 1971; English transl., Springer-Verlag, New York, 1974. MR 49 \#6287; 49 \#11603.

3. L. S. Grinblat, Compactifications of spaces of functions and integration of functionals, Trans. Amer. Math. Soc. 217 (1976), 195-223.

4. H. Schubert, Topology, Teubner, Stuttgart, 1964; English transl., Allyn and Bacon, Boston, Mass., 1968. MR 30 \#551; 37 \#2160.

Department of Mathematics, Bar-Ilan University, Ramat-Gan, Israel

Current address: Department of Mathematics, Harvard University, Cambridge, Massachusetts 02138 